\title{
A System For Landslides Monitoring Using Wireless Underground Sensor Networks and Cloud Computing
}

\author{
Ana Beatriz C. Pinho \\ CEFET/RJ-Campus Petrópolis \\ ana.cunha@aluno.cefet-rj.br
}

\author{
André Monteiro \\ CEFET/RJ-Campus Petrópolis \\ andre.monteiro@cefet-rj.br
}

\author{
Felipe Henriques \\ CEFET/RJ-Campus Petrópolis \\ felipe.henriques@cefet-rj.br
}

\begin{abstract}
This work presents a system for remote monitoring of landslides based on a wireless underground sensor network. The sensor network is implemented on Arduino components connected through Wi-Fi modules, and is responsible to collect soil moisture rates in real time. Then, the collected data is sent to a cloud computing environment in order to ensure a robust and secure storage. Moreover, the cloud platform enables data mining and the visualization of the information by the government and general people using an App designed for mobile devices. To analyze the feasibility of the system, real tests were performed using a prototype. The initial results show the sensor network accurately measured the soil moisture and the cloud computing platform was able to store and to display the collected data in real time properly to the users.
\end{abstract}

\section{KEYWORDS}

Sensor Network, Landslides Monitoring, Cloud Computing

\section{INTRODUCTION}

The landslides are an usual phenomenon in Brazil, due to the tropical weather with great amounts of rain, mostly on summer. Besides that, there are several mountainous terrain along the country which are occupied by legal and illegal buildings. These occupations increase the risk of landslides, once the original vegetation was removed from those areas. As shown in [1], in the year of 2010 over 8 million people, which live in 2.4 million of habitations located in 872 cities, remain in areas with potential risk of landslides.

Despite some states and cities present safety protocols to evacuate people in critical rainy scenarios, as can be seen in Rio de Janeiro's protocol described in [2], most of these action plans are not efficient and are based on simple approaches, such sirens of alert, for instance. Moreover, most of these approaches consider weather forecast information and pluviometric rates collected from rain sensors spread on different locations around the cities. More to the point, the data is not collected from hills and slopes mapped as risky areas, which leads to a ineffective model to predict landslides and to evacuate people as well.

This work address the landslides remote monitoring, and is based on a wireless underground sensor network to collect data directly from the target areas. To this end, humidity sensors are deployed in the soil of mountainous terrain previously mapped as risky areas by the local authorities. Then, in order to ensure a robust and secure storage, the data collected by the underground sensor network is sent to a cloud computing platform. The system also uses an App designed for mobile devices (smartphones and tablets) where the data could be displayed in real time using a friendly interface. In addition, the App could enable the sending of warning to people who live in risky areas that a landslide is about to occur.
The main goal of the proposed approach is to enable a real time monitoring of the soil moisture in risky areas by everyone interested (authorities and citizens). Furthermore, the cloud computing environment is powered by several prediction algorithms and tools based on IA. Therefore, the system is able to run in a proactive manner with a high level of accuracy, avoiding that people remain in risky areas when a landslide is about to begin. This is feasible once the data is directly collected from the soil of the moutainous terrain, processed and analyzed in real time.

The literature presents several works that address the remote soil monitoring based on underground sensor networks. Most of these approaches are designed for farming or general purpose activities, as shown in [3] [4]. Despite both works are aimed for farming, they have architectural similarities with our work, such as the Wi-Fi sensor network and the cloud computing platform for storing data. The works described in [5] and [6] present solutions for landslide monitoring. However, the main focus of these works are landslides caused by structural issues in constructions (dams, dikes, etc.), and use movement sensors for the remote monitoring process. As our work address landslides originated by the rain in moutainous terrains, the sensor network is aimed to monitor the soil moisture. Moreover, the mentioned works does not provide an App for public visualization in real time.

\section{THE PROPOSED MODEL}

As mentioned earlier, the proposed model uses a wireless underground sensor network with several humidity sensors deployed in the soil to be monitored. Then, the communication between the sensors and the access point is performed by the Wi-Fi protocol. The access point is responsible for sending the collected data to the cloud computing platform, where the data is processed and stored. Lastly, the cloud computing platform also has the role of a data server, feeding the App that runs on the mobile devices, which is responsible for data visualization and also for warning messages to the users when a landslide is about to occur. This architecture is shown in Fig. 1.

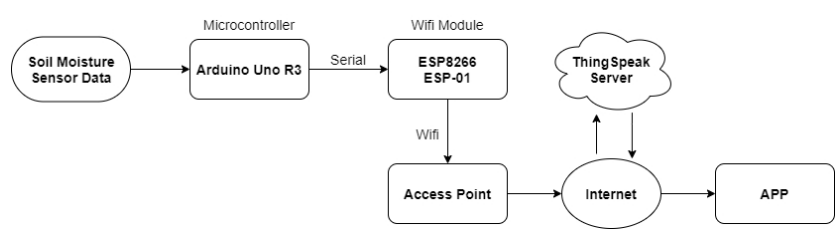

Figure 1: System architecture

A system prototype was implemented in this work using one Arduino Uno R3 attached with an humidity sensor. The Arduino 
uses a ESP8266 Wi-Fi module to send the collected data to the access point. Then, the access point routes the packets through the internet to a data channel previously configured in the ThingSpeak platform, which is a cloud computing environment that enables instant visualization of live data, data mining and alerts configuration, as described in [7].

The experiments performed was aimed to ratify the designed architecture. The testbed depicted in Fig. 2 was used to analyze if the humidity rates collected by the sensor would be properly stored and visualized in the cloud computing platform. To this end, an humidity sensor was deployed in a box with small amount of brown soil, which was filled with different amounts of water per time, simulating a rainy scenario and performing a similar approach with the work described in [8].

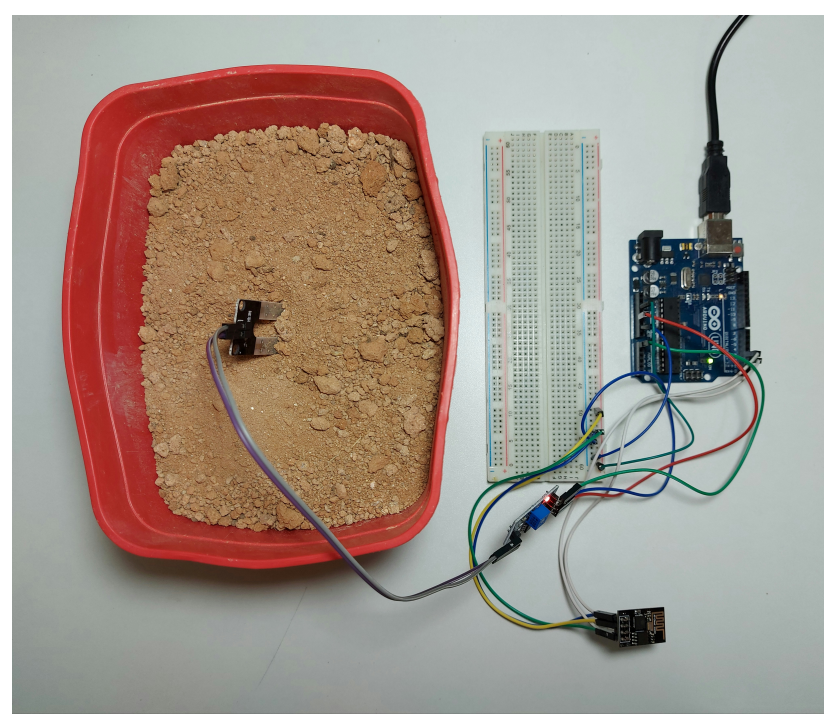

Figure 2: The prototype testbed

Every event related to fill the soil box with some amount of water uses the constant value of $10 \mathrm{ml}$ of water per time. In order to simulate different types of rainy scenarios, we choose three time intervals between each event. The first one is related to a light rain, and fills the soil box with $10 \mathrm{ml}$ of water every 6 minutes. The second scenario mapps a medium rain, and the $10 \mathrm{ml}$ of water is putted in soil box the every 3 minutes. Finally, to simulate a heavy rain, the amount of $10 \mathrm{ml}$ of water is placed in the soil box every 30 seconds.

For all the observed scenarios, the experiment was finished after 10 rounds, which means that the soil box was filled with $100 \mathrm{ml}$ of water. The value of the soil moisture rate is measured by the sensor at the end of the mentioned time intervals of each scenario. In other words, on the light rain scenario the first soil moisture rate is measured at 10 minutes, just before the second round of $10 \mathrm{ml}$ of water, the next measure occurs at 20 minutes, and so on. The results are presented in Fig. 3.

As expected, at the beggining of the experiment the highest soil moisture rate is related to the heavy rain scenario, since the measure was performed only 10 seconds later than the first round of $10 \mathrm{ml}$

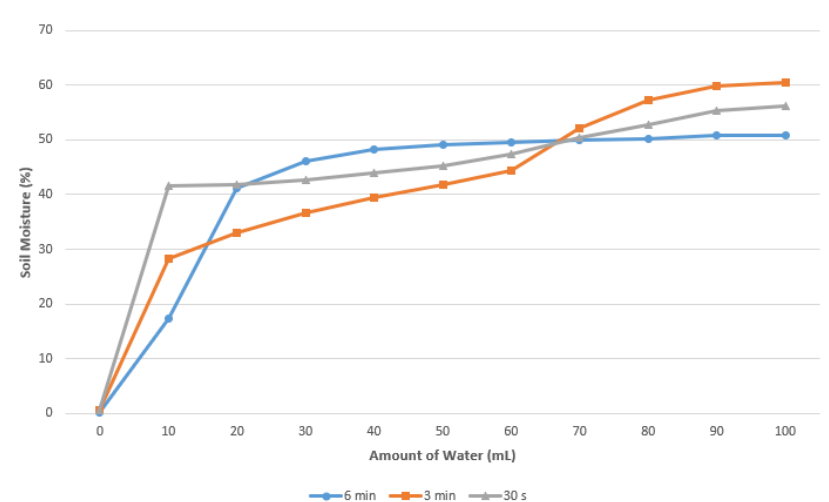

Figure 3: Results considering three rainy scenarios

of water. It is important to notice that around the seventh round of $10 \mathrm{ml}$ of water (totaling $70 \mathrm{ml}$ of water in the soil box) all the scenarios present a similar soil moisture rate. This point indicates the saturation of water in the soil box, once the new rounds of 10 $\mathrm{ml}$ of water have no relevant impact on the humidity rates collected by the sensor.

It is worth mentioning that each line in the graphic depicted in Fig. 3 was generated at runtime by the ThingSpeak platform. Then, all the collected data was combined into in a single chart to provide a better visualization in this paper and a holistic view of the soil moisture behavior on each rainy scenario. However, the ThingSpeak provides several data visualization options and a friendly control panel for personalized configuration according the user demand.

Once this proposed system is a work in progress, the App planned for data visualization and landslides warning in real time was not implemented yet, and is pointed as future work. Nevertheless, the visual concepts of the main App screens are presented in Fig. 4 with the GUI in Portuguese, since this project is initially addressed for people who live in Brazil. These main screens enable the real time monitoring of the soil moisture and the pluviometric rate from a selected location. In addiction, the screen designed for landslides warning is also presented.

As mentioned earlier, the soil moisture is obtained directly from the humidity sensors. Since the soil moisture and the pluviometric rate have a direct relationship, the latter could be calculated by analytic models, as shown in [9] and [10]. On the other hand, there are several public weather API available on the internet, such as Open Weather Map [11] and Free Weather API [12], which provide pluviometric rates and other relevant weather data from a specificc location with a suitable accuracy.

However, it is important to notice that even without an App to provide data visualization, the ThingSpeak platform was able to make feasible the real time monitoring of the soil moisture rates collected by the sensor network. This feature was the main reason that justify the ThinkSpeak as the cloud computing platform chosen for this project, since the authorities associated with civil defense activities could remote monitor selected areas only using the cloud computing interface. 


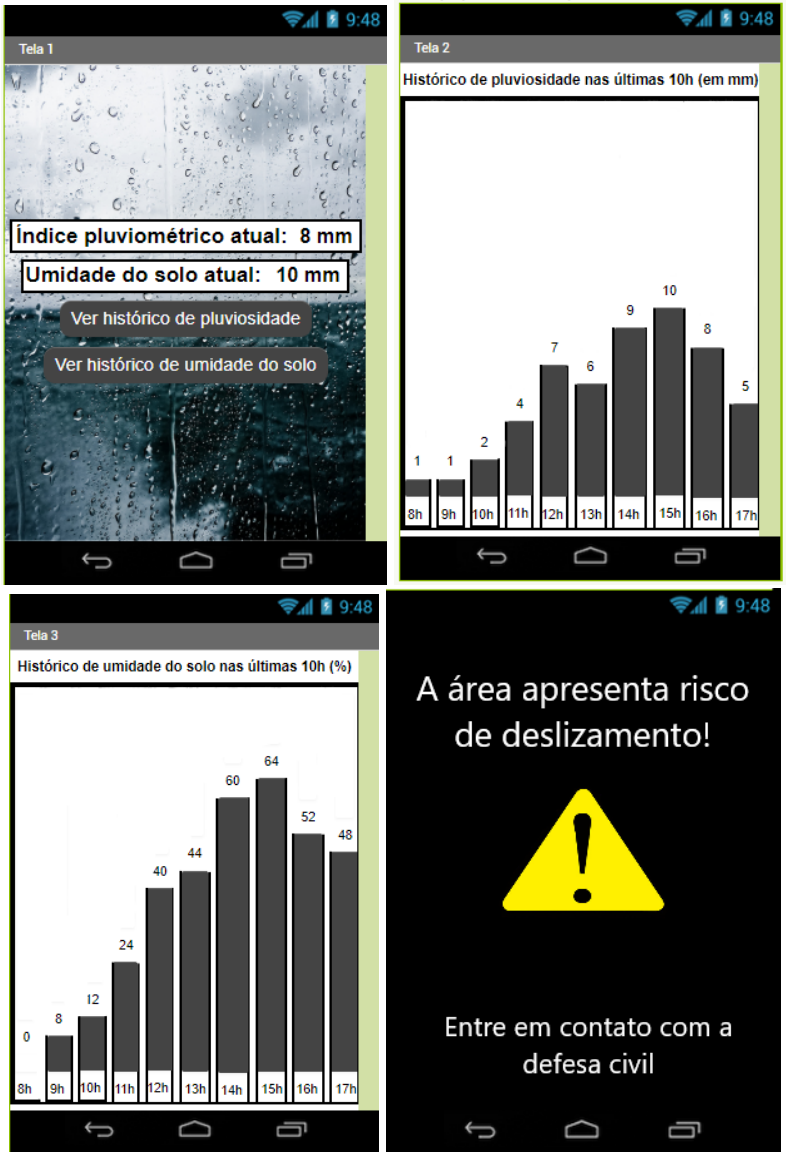

Figure 4: Visual concepts of the App screens

\section{CONCLUSION AND FUTURE WORK}

This work in progress presents a system for remote monitoring of landslides based on a wireless underground sensor network and a cloud computing platform. A prototype was implemented and evaluated through real tests, which was aimed to simulate rainy scenarios. The initial results ratify the model architecture, and show the system was able to measure the soil moisture rates with a suitable accuracy. Moreover, the cloud computing platform chosen for the system implementation enabled data visualization in real time, in addition to provide a reliable and robust storage for the data collected by the sensor network.

As future work, we intend to implement the App for mobile devices to provide the data visualization to the general public, and the warning of imminent landslides as well. Furthermore, we also intend to expand the underground sensor network in order to implement a mesh network, analyzing relevant issues related to this approach, such as power consumption of the wireless modules attached to the Arduino, network dependability, among others. More to the point, we will also analyze the efficiency of a hybrid wireless sensor network (underground and surface), adding rainfall sensors to the system architecture. To this end, we intend to evaluate the data transmission considering the main network protocols, such as
ZigBee, Bluetooth, LoRa, among others. This hybrid sensor network can be an interesting alternative to the use of the public weather API mentioned in the previous section, since the data collected from the rainfall sensors is more accurate than the provided by the weather API.

The warning process (metrics required for decision-making, triggers to warn the local population, etc.) will be also specified and evaluated. Lastly, the most suitable location of each sensor in the undergroud wireless network is a point of interest as well. Issues related to the depth of the sensor in the soil, data routing through the sensor network, and the optimal distance between each sensor will be explored considering the robustness of the sensor network and the trade-off between cost and performace

\section{REFERENCES}

[1] IBGE. População em áreas de risco no Brasil, 2018 (accessed July 19, 2020). URL https://agenciadenoticias.ibge.gov.br/agencia-sala-de-imprensa/2013-agenciade-noticias/releases/21565-em-2010-brasil-tinha-8-3-milhoes-de-pessoasmorando-em-areas-com-risco-de-desastres-naturais.

[2] Governo do Estado do Rio de Janeiro. Plano de Contingência para as Chuvas de Verão 2020/2021, 2021 (accessed February 19, 2021). URL https://agenciabrasil.ebc.com.br/geral/noticia/2020-12/governo-do-riode-janeiro-lanca-plano-para-enfrentar-chuvas-de-verao.

[3] R Deekshath, P Dharanya, Ms KR Dimpil Kabadia, Mr G Deepak Dinakaran, and $S$ Shanthini. Iot based environmental monitoring system using arduino uno and thingspeak. International fournal of Science Technology \& Engineering, 4(9), 2018.

[4] AM Ezhilazhahi and PTV Bhuvaneswari. Iot enabled plant soil moisture monitoring using wireless sensor networks. In 2017 Third International Conference on Sensing, Signal Processing and Security (ICSSS), pages 345-349. IEEE, 2017.

[5] Prapti Giri, Kam Ng, and William Phillips. Wireless sensor network system for landslide monitoring and warning. IEEE Transactions on Instrumentation and Measurement, 68(4):1210-1220, 2018.

[6] Vu Van Khoa and Shigeru Takayama. Wireless sensor network in landslide monitoring system with remote data management. Measurement, 118:214-229, 2018.

[7] The MathWorks Inc. ThingSpeak, 2020 (accessed August 3, 2020). URL http: //www.thingspeak.com.

[8] Creyton B de M Ferreira, Viviane F Peixoto, Jorge Augusto G de Brito, André Felipe de A Monteiro, Laura Silva de Assis, and Felipe da R Henriques. Underapp: A system for remote monitoring of landslides based on wireless underground sensor networks. In Anais Estendidos do XXV Simpósio Brasileiro de Sistemas Multimídia e Web, pages 79-82. SBC, 2019.

[9] Elfatih AB Eltahir. A soil moisture-rainfall feedback mechanism: 1. theory and observations. Water resources research, 34(4):765-776, 1998.

[10] Pablo Valenzuela, María José Domínguez-Cuesta, Manuel Antonio Mora García, and Montserrat Jiménez-Sánchez. Rainfall thresholds for the triggering of landslides considering previous soil moisture conditions (asturias, nw spain). Landslides, 15(2):273-282, 2018.

[11] Open Weather Map. Open Weather Map, 2020 (accessed October 22, 2020). URL https://openweathermap.org/.

[12] Free Weather API. Free Weather API, 2020 (accessed November 12, 2020). URL https://www.weatherapi.com/. 